\title{
Universality of the unitary Fermi gas: A few-body perspective
}

\author{
Jesper Levinsen, ${ }^{1}$ Pietro Massignan, ${ }^{2}$ Shimpei Endo, ${ }^{1}$ and Meera M. Parish ${ }^{1}$ \\ ${ }^{1}$ School of Physics and Astronomy, Monash University, Victoria 3800, Australia \\ ${ }^{2}$ ICFO-Institut de Ciencies Fotoniques, The Barcelona Institute of Science and Technology, 08860 Castelldefels, Spain
}

\begin{abstract}
We revisit the properties of the two-component Fermi gas with short-range interactions in three dimensions, in the limit where the $s$-wave scattering length diverges. Such a unitary Fermi gas possesses universal thermodynamic and dynamical observables that are independent of any interaction length scale. Focusing on trapped systems of $N$ fermions, where $N \leq 10$, we investigate how well we can determine the zero-temperature behavior of the many-body system from published few-body data on the ground-state energy and the contact. For the unpolarized case, we find that the Bertsch parameters extracted from trapped few-body systems all lie within $15 \%$ of the established value. Furthermore, the few-body values for the contact are well within the range of values determined in the literature for the many-body system. In the limit of large spin polarization, we obtain a similar accuracy for the polaron energy, and we estimate the polaron's effective mass from the dependence of its energy on $N$. We also compute an upper bound for the squared wave-function overlap between the unitary Fermi system and the non-interacting ground state, both for the trapped and uniform cases. This allows us to prove that the trapped unpolarized ground state at unitarity has zero overlap with its non-interacting counterpart in the many-body limit $N \rightarrow \infty$.
\end{abstract}

\section{INTRODUCTION}

Dilute gases of fermions with short-range interactions and diverging scattering lengths are of relevance to neutron stars, ultracold atomic gases, and possibly even to unconventional superconductors in the solid state $[1,2]$. Of particular interest is the two-component $(\uparrow, \downarrow)$ Fermi system, which has been shown to be stable in the unitarity limit where the $s$-wave scattering length $a \rightarrow \pm \infty[3-$ 5]. In this case, there is no interaction length scale at low energies, and the unitary Fermi gas displays a universal equation of state that only depends on the particle density, the spin polarization, and the temperature [6-10]. Moreover, the system is spatially scale invariant, which results in universal properties such as a vanishing bulk viscosity [11-13].

Despite its symmetry properties, the unitary manybody system is notoriously difficult to treat theoretically, since there is no small interaction parameter. Thus, a variety of sophisticated numerical methods have been used to attack the problem. The ground-state and finitetemperature thermodynamics of the unpolarized gas have been investigated using pairing fluctuations approaches, density-functional theory, and various types of quantum Monte Carlo (QMC) [14-17]. In the limit of large spin imbalance, where the system is well described by Fermi liquid theory, successful theoretical approaches include fixed-node and diagrammatic $\mathrm{QMC}$, variational wave functions, and ladder diagrams $[18,19]$.

Given that the unitary Fermi gas lacks a small interaction parameter, it is of interest to consider other perturbative approaches. A prominent example is the virial expansion, which is applicable in the regime where the system's temperature $T$ is far above quantum degeneracy. Taking advantage of the fact that the fugacity $z=e^{\mu / k_{B} T}$ is small in this limit, the entire thermodynamics may be determined from an expansion of the thermodynamic po- tential

$$
\Omega=-k_{B} T \frac{2 V}{\lambda^{3}} \sum_{N \geq 1} b_{N} z^{N}
$$

Here, $V$ is the volume, $k_{B}$ the Boltzmann constant, $\lambda$ the thermal wavelength, and $\mu$ the chemical potential, where we have considered the unpolarized system for concreteness. Crucially, the thermodynamic potential depends only on few-body correlations through the virial coefficients $b_{N}$ [20-23], which are completely determined from the energy spectrum of $N$ particles. Recent cold-atom experiments on the thermodynamics of the unitary Fermi gas $[9,24]$ have observed very good agreement with the results from the virial expansion, even for a weakly degenerate gas. For a review of the virial expansion, we refer the reader to Ref. [25].

In this paper, we consider the opposite limit and ask whether few-body physics can also be used to gain insight into the behavior of a strongly interacting unitary Fermi gas at zero temperature. Our approach is particularly motivated by recent work on the harmonically trapped one-dimensional Fermi gas. Here, the Heidelberg experiment led by S. Jochim explored the evolution from few- to many-body physics using a single $\downarrow$ impurity atom repulsively interacting with $N_{\uparrow}$ spin- $\uparrow$ fermions, where $N_{\uparrow}$ was increased from 1 to 5 [26]. In this few-body system, they observed a fast convergence of the interaction energy towards the expected many-body result [27]. Subsequently, it has been predicted that both the energy and contact of a spin-balanced gas in a one-dimensional harmonic trap converge rapidly towards the many-body limit, for any interaction strength [28]. Thus, this raises the question of whether few-body correlations can similarly determine the behavior of the three-dimensional unitary Fermi gas.

To investigate the relationship between the few- and many-body systems at unitarity, we analyse data from trapped systems of $N$ equal-mass fermions, where $N \leq$ 10. Unless otherwise specified, the results we quote arise 
from calculations for the unitary harmonically trapped Fermi gas. For a comprehensive review of few-body physics in harmonic traps, we refer the reader to Ref. [29]. We focus on static quantities at zero temperature such as the energy, the contact, and the squared wave-function overlap with the non-interacting state. Furthermore, we only consider the ground state of the system and we ignore the behavior of the metastable repulsive branch, such as the possibility of itinerant ferromagnetism [30].

We emphasize that our primary objective is not to provide precise predictions for the many-body properties of the unitary Fermi gas. Rather, we will determine which of these properties may be reasonably well described within few-body calculations, and whether these properties converge quickly towards the many-body limit. We also discuss how the squared wave-function overlap between the unitary and non-interacting ground states evolves with particle number $N$, and we calculate an upper bound for this overlap for general $N$.

The manuscript is organized as follows. In Section II we review the fundamental properties of the uniform unitary Fermi gas, focussing on equal spin populations and large spin imbalance, and we introduce the local density approximation for trapped systems of particle number $N \gg 1$. In Section III we show how one may obtain surprisingly accurate predictions for the energy of the unitary Fermi gas in the thermodynamic limit from a knowledge of the energies of very few trapped atoms $(2 \leq N \leq 10)$. The related analysis presented in Section IV once again leads to an extrapolated contact which lies well within the range of the most recent theoretical and experimental determinations. In Section V we consider the overlaps between the non-interacting and strongly interacting wave functions. Here we derive a rigorous upper bound of this overlap and we determine a general relationship between the overlap in the trapped system and that in uniform space. We conclude in Section VI, by presenting an outlook, and by summarizing our main results.

\section{THE UNITARY FERMI GAS}

We begin our study of the unitary Fermi gas by considering the universal properties of the many-body system with spin components $\sigma=\uparrow, \downarrow$, where the total number of fermions $N=N_{\uparrow}+N_{\downarrow} \gg 1$. To simplify the discussion, we focus on the equal-mass case, where $m_{\uparrow}=m_{\downarrow} \equiv m$, and we work in units where $\hbar=k_{B}=1$.

For a uniform system at temperature $T$ and in a volume $V$, the general equation of state for the total energy $E$ can be written as:

$$
E=\frac{3}{5} \frac{k_{F}^{2}}{2 m} N \beta\left(\frac{T}{E_{F}}, \frac{N_{\downarrow}}{N_{\uparrow}}, \frac{1}{k_{F} a}\right) .
$$

Here, we assume that $N_{\downarrow} \leq N_{\uparrow}$, without loss of generality, and we define the Fermi momentum $k_{F}=\left(6 \pi^{2} N_{\uparrow} / V\right)^{1 / 3}$ and Fermi energy $E_{F}=k_{F}^{2} / 2 m$. We neglect the microscopic details of the interaction, such as the range $r_{0}$, since we are considering the regime of low-energy resonant scattering, $k_{F}\left|r_{0}\right| \ll 1$ and $\left|r_{0}\right| \ll|a|$.

Taking the limits $1 / a \rightarrow 0$ and $T \rightarrow 0$, it is clear that Eq. (1) becomes a universal function of the particle numbers $N_{\uparrow}, N_{\downarrow}$. In particular, when the system is unpolarized, i.e., $N_{\uparrow}=N_{\downarrow}$, we obtain the universal Bertsch parameter: $\xi \equiv \beta(0,1,0)[3]$. In the opposite limit of a large spin polarization, $N_{\downarrow} / N_{\uparrow} \rightarrow 0$, we have $\beta \rightarrow 1$.

One can fully characterize the universal thermodynamics of the uniform unitary Fermi gas at zero temperature from just three observables: the energy $E$, the contact

$$
C=-4 \pi m\left(\frac{\partial E}{\partial a^{-1}}\right)_{N_{\uparrow}, N_{\downarrow}},
$$

and the (inverse) spin susceptibility

$$
\chi_{s}^{-1}=V\left(\frac{\partial^{2} E}{\partial M^{2}}\right)_{N},
$$

where the magnetization $M=N_{\uparrow}-N_{\downarrow}$. In principle, there are other static observables such as the compressibility $\kappa$ and the heat capacity $\mathcal{K}_{V}$, but $\mathcal{K}_{V} \rightarrow 0$ in the limit of zero temperature, while the compressibility is simply related to the energy: $\kappa^{-1}=\frac{10}{9} \frac{E}{V}$. We will not concern ourselves with dynamical (transport) quantities like the viscosity [11-13] or conductivity. These also have interesting symmetry properties, but are non-trivial to extract from the few-body system.

\section{A. Unpolarized gas}

The behaviour of the unitary Fermi gas at zero temperature simplifies considerably when $N_{\uparrow}=N_{\downarrow}$. In this case, the energy in Eq. (1) reduces to

$$
E=\xi \frac{3}{5} \frac{k_{F}^{2}}{2 m} N \equiv \xi E_{0},
$$

where $E_{0}$ is the energy of the non-interacting system. Thus, the thermodynamic quantities that directly follow from Eq. (4) only depend on the particle density and the Bertsch parameter. Since the ground state of the unpolarized system is a paired superfluid, another quantity of interest is the pairing gap $\Delta$. This is the minimum energy required to add an unbound quasiparticle and is thus a feature of the quasiparticle excitation spectrum. The existence of such a gap results in an exponentially vanishing spin susceptibility as $T \rightarrow 0$ [31].

However, it is generally not straightforward to relate the pairing gap in the trapped gas to that in the uniform system. While one can define an excitation gap for having an unbound particle in the trapped system [29], this extra particle becomes confined to the trap edge as the number of particles $N \rightarrow \infty$. Thus, it will sensitively depend on the system boundary and it cannot be considered a "bulk" property. Indeed, even experiments on 
trapped ultracold atomic gases must perform local radiofrequency spectroscopy near the trap center in order to extract the pairing gap for a uniform system [32].

To define a bulk pairing gap for the trapped gas, one can analyse the spatially varying pairing field within mean-field theory [33]. This analysis would suggest that we have the local contact density $C / V=m^{2}\left(\Delta^{2}+U^{2}\right)$ at every point in the trap, where $U$ is the Hartree energy shift in the quasiparticle spectrum [34]. Using the experimental observation that $U \approx \Delta$ at unitarity [32], we then obtain $C / V \approx 2 m^{2} \Delta^{2}$. Therefore, we will use the contact as a measure of the pairing gap in the trapped unitary system.

\section{B. Limit of large spin polarization}

When there is a small concentration of spin- $\downarrow$ atoms, $x=N_{\downarrow} / N_{\uparrow} \ll 1$, the system can be described in terms of dressed impurities, or "polarons", immersed in an ideal Fermi sea. Such polarons are weakly interacting quasiparticles characterised by three parameters: the impurity energy $E_{\text {pol }}$ at zero momentum, the effective mass $m^{*}$, and the residue $Z$ (defined as the squared overlap between the interacting and non-interacting ground-state wave functions). For a comprehensive survey of the vast literature on Fermi polarons, we refer the reader to recent reviews covering the subject $[18,19,35]$.

These basic quasiparticle properties define the zerotemperature equation of state for the polarized Fermi gas, which takes the Landau-Pomeranchuk form [36, 37]

$$
E=\frac{3}{5} E_{F} N_{\uparrow}+\left(E_{\mathrm{pol}}+\frac{3}{5} E_{F} \frac{m}{m^{*}} x^{2 / 3}\right) N_{\downarrow}
$$

in the limit $x \ll 1$. The terms on the right hand side of Eq. (5) correspond to, respectively, the energy of the unperturbed majority Fermi sea, the energy of the impurities dressed by the majority atoms, and the Fermi pressure associated with the finite concentration of dressed impurities. At this level of approximation, the polarons behave as ideal quasiparticles, since the interactions between polarons contribute only at order $x$ to the system's energy [37].

From Eqs. (3) and (5), the spin susceptibility in this limit is

$$
\chi=x^{1 / 3} \frac{6 m^{*}}{E_{F} m} \frac{N_{\uparrow}}{V} \equiv \frac{m^{*}}{m} \chi_{0},
$$

where $\chi_{0}$ is the spin susceptibility of the non-interacting Fermi gas in the ground state. Thus, $\chi$ provides a direct probe of the effective mass of the polaron.

In a uniform system, the quasiparticle properties can be accurately described within a simple variational approach [38], where only a single particle-hole excitation of the Fermi sea is included. Specifically, for a polaron at zero momentum, the wave function within the one- particle-hole (1PH) Ansatz reads

$$
\left|\psi_{\mathbf{0}}\right\rangle=\phi_{0} c_{\mathbf{0} \downarrow}^{\dagger}|F S\rangle_{\uparrow}+\sum_{q<k_{F}}^{k>k_{F}} \phi_{\mathbf{k q}} c_{\mathbf{q}-\mathbf{k}_{\downarrow}}^{\dagger} c_{\mathbf{k} \uparrow}^{\dagger} c_{\mathbf{q} \uparrow}|F S\rangle_{\uparrow},
$$

where $c_{\mathbf{p} \sigma}$ annihilates a particle with spin $\sigma$ and momentum $\mathbf{p},|F S\rangle_{\uparrow}$ is the ground-state of the non-interacting spin- $\uparrow$ Fermi sea, and $\phi_{0}, \phi_{\mathbf{k q}}$ are variational parameters. Minimizing the total energy of the system leads to a selfconsistent equation of the form $E_{\mathrm{pol}}=\Sigma\left(\mathbf{p}=\mathbf{0}, E_{\mathrm{pol}}\right)$ [38], which is formally equivalent to finding the real pole of the dressed impurity Green's function [39]

$$
G_{\downarrow}(\mathbf{p}, \omega)=\frac{1}{\omega-\varepsilon_{\mathbf{p}}-\Sigma(\mathbf{p}, \omega)+i 0_{+}} .
$$

Here $\varepsilon_{\mathbf{p}}=p^{2} / 2 m$, and $\Sigma$ is the retarded impurity self-energy computed within the T-matrix (or "forwardscattering") approximation, which at zero momentum is given by

$$
\begin{aligned}
& \Sigma(\mathbf{0}, \omega)=\sum_{q<k_{F}}\left[\frac{m}{4 \pi a}\right. \\
& \left.-\sum_{k>k_{F}}\left(\frac{1}{\omega-\left(\varepsilon_{\mathbf{q}-\mathbf{k}}+\varepsilon_{k}-\varepsilon_{q}\right)+i 0_{+}}+\frac{m}{k^{2}}\right)\right]^{-1} .
\end{aligned}
$$

Using this approach, one finds $E_{\text {pol }} \approx-0.61 E_{F}, Z \approx$ 0.78 , and $m^{*} \approx 1.17 m$. These values compare remarkably well with the results of state-of-the-art calculations: $E_{\mathrm{pol}}=-0.615 E_{F}, Z=0.759$, and $m^{*}=1.197 m[40-42]$.

The fact that one obtains such excellent results from a simple Ansatz, which only accounts for two-body correlations exactly, motivates us to analyze the quasiparticle properties from the few-body limit.

\section{Trapped system and the local density approximation}

To connect with the trapped few-body system, we must consider the Fermi gas in the presence of an isotropic harmonic potential $V(\mathbf{r})=\frac{1}{2} m \omega^{2} r^{2}$, where $\omega$ is the trapping frequency. We implicitly assume that the harmonic oscillator length $a_{\mathrm{ho}} \equiv 1 / \sqrt{m \omega}$ is much larger than the range $\left|r_{0}\right|$, so that we remain in the unitary regime.

In the limit where the total number of particles $N \gg 1$, the local density approximation (LDA) holds [43] and we may determine global quantities from averages over local quantities in the trap, i.e., for a local observable $F$, we have trapped observable $\mathcal{F}=\frac{1}{V} \int d^{3} r F(\mu(\mathbf{r}))$ [90]. Here, we define the local chemical potential $\mu(\mathbf{r})=\mu-V(\mathbf{r})$, which is connected to the global chemical potential

$$
\mu=\frac{\partial \mathcal{E}}{\partial N},
$$

where $\mathcal{E}$ is the total energy of the trapped gas. 
For a trapped, non-interacting Fermi gas with balanced spin populations, we have particle density

$$
n(\mathbf{r})=\frac{1}{3 \pi^{2}}(2 m)^{3 / 2}\left(\mu-\frac{1}{2} m \omega^{2} r^{2}\right)^{3 / 2},
$$

thus giving the total number of particles

$$
N=N_{\uparrow}+N_{\downarrow}=\int d^{3} r n(\mathbf{r})=\frac{\mu^{3}}{3 \omega^{3}} .
$$

Similarly to the uniform system, we can use the relationship between particle number and $\mu$ in Eq. (12) to define a Fermi momentum and Fermi energy:

$$
\kappa_{F}=\left(48 N_{\uparrow}\right)^{1 / 6} a_{\mathrm{ho}}^{-1}, \quad \varepsilon_{F}=\kappa_{F}^{2} / 2 m .
$$

These quantities are equivalent to the local Fermi momentum and Fermi energy at the center of the trap.

From Eq. (10), we find the energy of the noninteracting trapped system to be

$$
\mathcal{E}_{0}=\int_{0}^{N} d N^{\prime} \mu\left(N^{\prime}\right)=\frac{\omega}{4}(3 N)^{4 / 3}=2 \mathcal{E}_{0 \uparrow}
$$

where the energy of the spin- $\uparrow$ component is given by:

$$
\mathcal{E}_{0 \uparrow}=\frac{\omega}{8}\left(6 N_{\uparrow}\right)^{4 / 3} \text {. }
$$

Note that one cannot directly average the energy over the trap, since it is not a function of the local chemical potential. However, one can perform a trap average on the thermodynamic potential $\Omega(\mu, h)=E-\mu N-h M$, where $h$ is an effective Zeeman field.

In the presence of interactions, the contact of the trapped system is [29]

$$
\mathcal{C}=-4 \pi m\left(\frac{\partial \mathcal{E}}{\partial a^{-1}}\right)_{N_{\uparrow}, N_{\downarrow}}
$$

which is equivalent to a trap averaged contact within LDA since we can write

$$
\mathcal{C}=-\frac{4 \pi m}{V} \int d^{3} r \frac{\partial \Omega(\mu(\mathbf{r}), h)}{\partial a^{-1}}=\int d^{3} r \frac{C(\mu(\mathbf{r}))}{V} .
$$

Here, we have used the fact that the contact can be defined from either $E$ or $\Omega$ [44].

For the unitary unpolarized Fermi gas, we see from Eq. (4) that we must simply replace $\mu(\mathbf{r})$ by $\xi^{-1} \mu(\mathbf{r})$ in Eq. (11), thus giving

$$
N=\frac{\mu^{3}}{3 \omega^{3} \xi^{3 / 2}}, \quad \mathcal{E}=\sqrt{\xi} \mathcal{E}_{0} .
$$

Furthermore, the dimensionless trap averaged contact is $[45,46]$

$$
\frac{\mathcal{C}}{N \kappa_{F}}=\frac{256}{105 \pi \xi^{1 / 4}}\left(\frac{C}{N k_{F}}\right),
$$

where the dimensionless contact for the unpolarized uniform system at unitarity is given by

$$
\frac{C}{N k_{F}}=-\left.\frac{6 \pi}{5} \frac{\partial \beta(0,1, y)}{\partial y}\right|_{y=0} .
$$

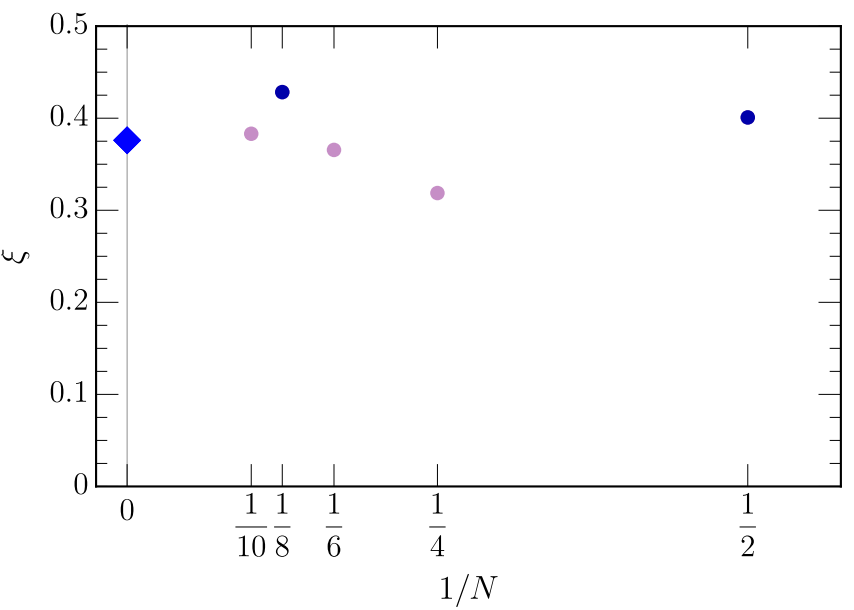

FIG. 1: Bertsch parameter of a balanced unitary Fermi gas calculated according to Eq. (21) (filled circles), using the energies for $N$ trapped fermions reported in Refs. [47, 48]. The error bars resulting from uncertainty in the few-body energies are smaller than the symbol sizes. The darker circles indicate closed shells of the corresponding non-interacting problem in the harmonic oscillator. The diamond shows the experimental data point [9], which has an error bar smaller than the symbol size.

\section{ENERGY AND THE BERTSCH PARAMETER}

The energy of a few harmonically trapped fermions in the unitary limit has been accurately predicted in a series of works, starting with the exact solution by Busch et al. for the two-spin $\uparrow \downarrow$ problem [47]. Here, it was shown that the energy at unitarity is exactly $2 \omega$; that is, interactions have reduced the energy from its value of $3 \omega$ in the limit of weak attractive interactions, $1 / a \rightarrow-\infty$. This reduction of the total energy upon approaching unitarity is a generic feature, which is reflected in the Bertsch parameter of the unpolarized many-body system, and in the polaron energy, as we discuss in this section.

In the following, we focus on data for the ground-state energy from precise few-body calculations [21, 47-53]. This presents an alternative angle to more intensive numerical studies of many trapped particles in the spinbalanced case [54-61] and for large spin polarizations [62].

In the limit $N \gg 1$, we know from LDA that the Bertsch parameter relates the energy of the unitary Fermi gas to that of the non-interacting system through $\sqrt{\xi}=$ $\mathcal{E} / \mathcal{E}_{0}$ - see Eq. (18). In order to minimize shell effects for smaller $N$, it is convenient to think of the Bertsch parameter as arising from an interaction energy shift compared with the non-interacting limit $1 / a \rightarrow-\infty$. That is, we measure the energy at unitarity with respect to the exact non-interacting energy $\mathcal{E}_{\mathrm{NI}}$ of the few-body system. We then normalize this shift by the non-interacting energy (14) calculated within LDA, as this does not contain any 
shell effects. In this manner, we modify Eq. (18) to give

$$
\xi=\left[1+\left(\mathcal{E}-\mathcal{E}_{\mathrm{NI}}\right) / \mathcal{E}_{0}\right]^{2},
$$

which, of course, converges to Eq. (18) when $N \rightarrow \infty$.

The result of our analysis is shown in Fig. 1. We see that the Bertsch parameter obtained within few-body calculations yields a value that is remarkably close to (within 15\% of) the value reported in experiment [9]

$$
\xi=0.376(4)
$$

and corroborated by QMC calculations [63]. Even the simplest result, given by the exact two-body solution, has a relative error of only about $7 \%$, further supporting the central idea that the Bertsch parameter is primarily determined by two-body correlations in the gas.

Let us now turn to the high polarization limit, where $N_{\downarrow}=1$. In this case, the interaction energy shift at unitarity can be related to the polaron energy in uniform space. Most notably, it has been predicted both in 3D [62] and in 1D [64] that the trapped density distribution of the impurity in the many-body limit is almost unaffected by strong interactions. In other words, the impurity is always confined to the center of the trap, even in the presence of a medium. Consequently, we expect that the polaron interaction energy is proportional to the chemical potential of the majority fermions at the center of the trap, i.e.,

$$
E_{\mathrm{pol}}=\mathcal{E}-\mathcal{E}_{\mathrm{NI}}=-A \varepsilon_{F},
$$

where we again use $\mathcal{E}_{\mathrm{NI}}$ as the exact energy of the noninteracting system. The coefficient of proportionality $A$ should asymptote towards the same value as in the uniform system, $E_{\text {pol }}=-0.615 E_{F}[42]$.

In Fig. 2, we show the polaron energy obtained in this manner from few-body calculations of the energy. The figure explicitly shows that only very few spin- $\uparrow$ particles are needed to obtain an approximate value of the polaron energy. In particular, from the exact solution of the two-body problem, we find $E_{\text {pol }}=-6^{-1 / 3} \varepsilon_{F} \approx-0.55 \varepsilon_{F}$ which has a relative error of only about $10 \%$ compared with the $N_{\uparrow} \rightarrow \infty$ limit. We note that a related analysis was carried out by D. Blume [62].

The few-body results for the ground-state energy also allow us to estimate the polaron effective mass [36]. Within LDA, the effective trapping potential experienced by the polaron is approximately [65]

$$
V_{\mathrm{eff}}(\mathbf{r})=\frac{1}{2} m \omega^{2} r^{2}-A \mu(\mathbf{r})=\frac{1}{2} m(1+A) \omega^{2} r^{2}-A \varepsilon_{F},
$$

and thus the ground-state energy of a particle of mass $m^{*}$ takes the form $\frac{3}{2} \sqrt{\frac{m}{m^{*}}(1+A)} \omega-A \varepsilon_{F}$. Using the relation between the Fermi energy and the particle number in Eq. (13), we thus arrive at

$$
\frac{E_{\mathrm{pol}}}{\varepsilon_{F}}=-A+\frac{3}{2} \sqrt{\frac{m}{m^{*}}(1+A)}\left(6 N_{\uparrow}\right)^{-1 / 3} .
$$

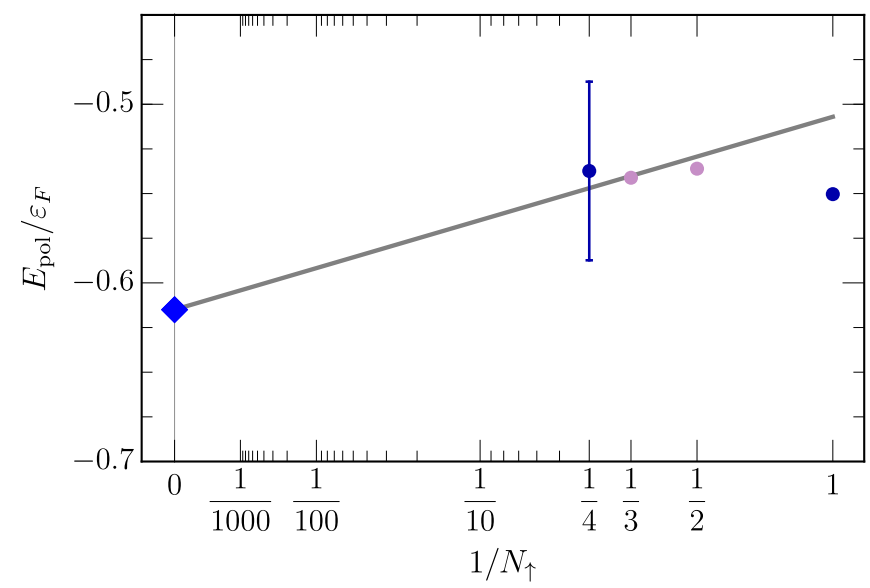

FIG. 2: Polaron energy (circles) in units of the local spin- $\uparrow$ Fermi energy at the center of the trap as a function of particle number $N_{\uparrow}$. The darker circles indicate closed shells. In the many-body limit of $N_{\uparrow} \rightarrow \infty$, the polaron energy is expected to approach the uniform system result, $E_{\mathrm{pol}}=-0.615 E_{F}$ [42], indicated as a diamond. The few-body results are taken from Refs. [21, 47, 49, 51]. The straight line is a fit to the data points for 2-4 majority particles using the form Eq. (24), keeping the known coefficient in the many-body limit fixed to $A=0.615$.

This demonstrates that the leading order correction to the $N_{\uparrow} \rightarrow \infty$ limit for the polaron energy goes like $N_{\uparrow}^{-1 / 3}$ in the trapped system, as illustrated in Fig. 2. By fitting Eq. (24) to the data (see Fig. 2), we obtain an effective polaron mass of $m^{*}=1.26 m$, which is in reasonable agreement with the result of QMC calculations, where $m^{*}=1.197 m[42]$.

\section{CONTACT}

A quantity that is naturally associated with two-body physics is the contact $[66,67]$. As defined in Eq. (2), it is intrinsically a thermodynamic quantity. However, it can also be related to the probability of finding two particles at a separation much less than the average interparticle spacing, $C=16 \pi^{2} \lim _{\mathbf{r} \rightarrow \mathbf{0}} r^{2}\left\langle n_{\downarrow}(\mathbf{r} / 2) n_{\uparrow}(\mathbf{r} / 2)\right\rangle$, with $n_{\sigma}(\mathbf{r})$ the density of spin $\sigma$ particles at position $\mathbf{r}$ [68]. This expression exposes the two-body nature of the contact, even in a medium, and we now investigate how fewbody results for the contact compare with the many-body limit.

In the harmonically trapped system at unitarity, we may again obtain the contact analytically for the twobody problem from the exact solution [47], yielding $\mathcal{C}=$ $4 \pi \sqrt{2 / \pi} a_{\mathrm{ho}}^{-1}$. For larger spin-balanced gases, the trap averaged contact has been computed in few-body calculations in Ref. [48]. These results may be related to the contact in uniform space by applying the LDA — see Eq. (19).

In Fig. 3, we show the resulting contact converted 


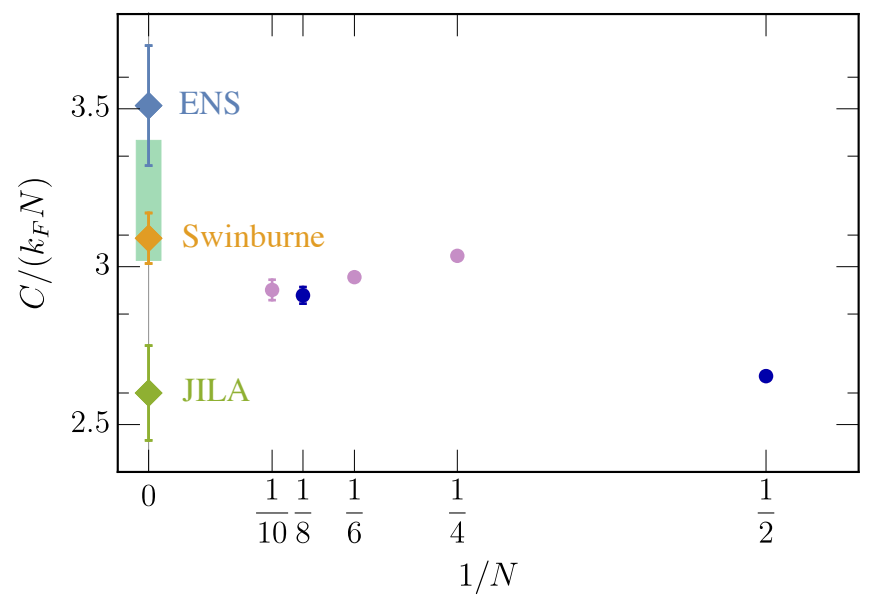

FIG. 3: Contact of the unpolarized unitary Fermi gas with $N$ particles. The few-body results $[47,48]$ are indicated by circles, where the darker circles denote closed shells. The experimental points (diamonds) are from Refs. [7, 46, 69]. The light green shading indicates the region of various recent theoretical results [46, 70-74].

to the uniform system. We see that the few-body results compare well with recent low-temperature measurements: Experiments at the ENS found $C /\left(N k_{F}\right)=$ $3.51 \pm 0.19[7,68]$, while the Swinburne group measured $C /\left(N k_{F}\right)=3.09 \pm 0.08$ [46]. At a somewhat higher temperature $\left(T \sim 0.16 T_{F}\right)$, the JILA group found $C /\left(N k_{F}\right) \approx 2.6 \pm 0.15$ [69]. We also note that numerous authors have evaluated the contact theoretically, finding values of $C /\left(N k_{F}\right)$ ranging from 3.01 to $3.40[46,70-74]$ (see, e.g., Ref. [74] for an extensive review). These values are all compatible with the few-body results.

From our results for the contact, we can also estimate the value of the pairing gap using the expression $C / V \approx 2 m^{2} \Delta^{2}$ from Sec. II A. Taking $C /\left(k_{F} N\right) \approx 3$ in the many-body limit, we obtain $\Delta / E_{F} \approx \sqrt{2 / \pi^{2}} \approx 0.45$, which compares remarkably well with the experimentally determined value of $\Delta / E_{F} \approx 0.44$ [32]. While this is certainly not a formal proof, it does raise the question of whether the contact at unitarity is intimately connected to the pairing gap.

Turning now to the polaron limit, we again take advantage of how the impurity is primarily located at the center of the trap. Therefore, in this case, we derive the uniform contact by simply equating the local Fermi momentum $\kappa_{F}$ at the center of the trap with its uniform space counterpart, $k_{F}$. Moreover, from Eq. (24), we see that the leading order correction to the many-body limit once again scales as $N_{\uparrow}^{-1 / 3}$ in the trapped system. In Fig. 4 we show the results of few-body calculations for the trapped contact [75], where we have converted the results using the relation between $\kappa_{F}$ and the majority particle number, Eq. (13). We see that the few-body contact takes values ranging between $4 \kappa_{F}$ and $6 \kappa_{F}$. In this case, the contact has not been measured experimentally, however, it has been calculated within the ansatz described in Sec-

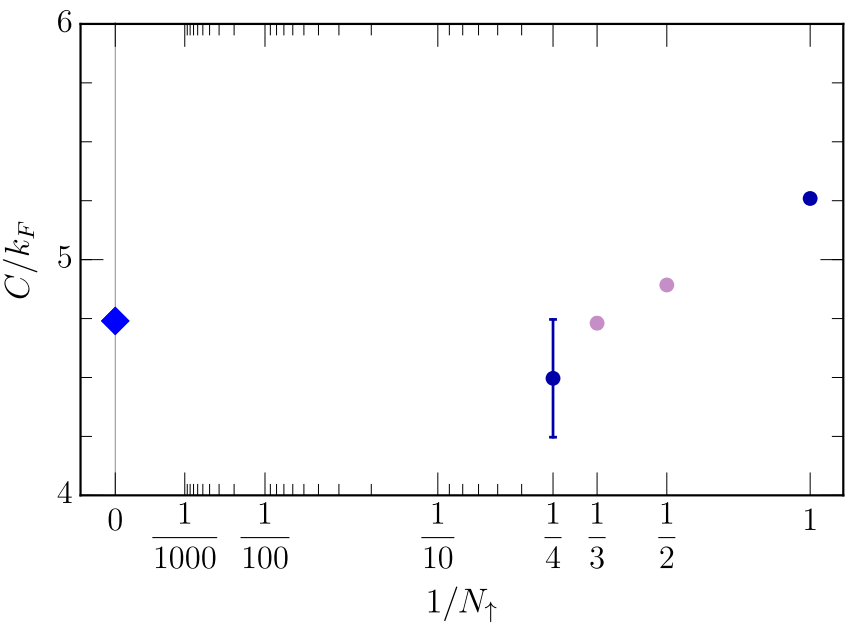

FIG. 4: Contact of a single $\downarrow$ impurity in a gas with $N_{\uparrow}$ majority fermions. The few-body results $[47-49,75,76]$ are marked with circles, the darker circles corresponding to closed shells. The diamond indicates the thermodynamic result in uniform space obtained from the 1PH Ansatz [77].

tion II B to be $C=4.74 k_{F}$ [77]. Given that this ansatz produces very reliable results for the other quasiparticle parameters, there is no a priori reason to believe that this result is inaccurate. The agreement with the results from the harmonically trapped few-body calculation is also quite reasonable.

\section{WAVE-FUNCTION OVERLAPS}

The squared wave-function overlap between a strongly interacting state and its non-interacting counterpart is an important quantity in many-body physics. For instance, in the case of the polaron, this overlap gives its quasiparticle weight, or residue. The overlap can be measured in Rabi oscillations as a shift of the oscillation frequency [78-80]. While the polaron residue of the $N_{\uparrow}+1$ system (a system of $N_{\uparrow}$ spin $\uparrow$ fermions and one spin $\downarrow$ impurity) in the many-body limit $N_{\uparrow} \rightarrow \infty$ has been actively studied both experimentally [78, 80, 81] and theoretically [77], it is still an open question how the polaron residue can be understood from a few-body perspective.

For two-particle systems, the "residue" of the groundstate wave function at unitarity is obtained analytically as

$$
Z=\frac{3}{4} \text { (uniform); } \mathcal{Z}=\frac{2}{\pi} \simeq 0.64 \quad(\text { trap }) .
$$

The uniform system result can be calculated by noting that the relative wave function of two non-interacting particles at zero energy is a constant, while for two particles at unitarity it is proportional to $1 / r$. In a large sphere of volume $V=4 \pi L^{3} / 3$, we then find The trapped result in Eq. (25) can be similarly obtained from the analytic solution of the two-body problem in a harmonic 
trap [47]. However, such exact calculations will be exponentially more challenging as the number of particles increases.

In this section, we use a hyper-spherical coordinates method to study the squared overlap between the ground state wave function of the unitary Fermi gas and its noninteracting counterpart in a harmonic trap as well as in a uniform system. We present a general formalism to calculate it for arbitrary particle numbers based on the separability of the hyper-radial and hyper-angular parts of the wave functions in the unitary and non-interacting systems. We derive a rigorous upper bound of the squared overlap, $Z_{>} \geq Z$, as a function of the energy of the trapped unitary and non-interacting systems. We evaluate this for systems with $N_{\uparrow}, N_{\downarrow} \lesssim 5$ using energies of the few-body unitary Fermi gas in a harmonic trap [21, 48], and then extend the calculation to $N \gg 1$ using LDA. We find that our rigorous upper bound for the polaron system approaches $Z_{>} \rightarrow 1$ as $N_{\uparrow} \rightarrow \infty$ for both trapped and uniform systems and thus does not actually provide a bound. On the other hand, the upper bound decreases exponentially, approaching $\mathcal{Z}_{>} \rightarrow 0$ for a trapped balanced system, dictating that the squared overlap must vanish in the many-body limit in the trap. Conversely, we find that it approaches a finite value $Z_{>} \rightarrow 0.942 \ldots$ in the uniform system.

\section{A. Hyper-spherical method to evaluate the squared overlap of the wave functions}

\section{Harmonically trapped systems}

For any $N_{\downarrow}+N_{\uparrow}$ system in an isotropic harmonic trap with unitary zero-range interactions, the wave function of $N$ particles at positions $\mathbf{r}_{i}, i=1, \cdots, N$, separates into center-of-mass, hyper-radial, and hyper-angular motions [12]:

$$
\Psi=\psi_{\mathrm{CM}}\left(\boldsymbol{R}_{\mathrm{CM}}\right) \frac{\mathcal{F}_{s}(R)}{R^{\frac{3 N-4}{2}}} \Phi_{s}(\Omega) .
$$

Here, $\boldsymbol{R}_{\mathrm{CM}}=\frac{1}{N} \sum_{i=1}^{N} \boldsymbol{r}_{\boldsymbol{i}}$ is the center-of-mass coordinate, $R^{2}=\sum_{i=1}^{N}\left(\boldsymbol{r}_{\boldsymbol{i}}-\boldsymbol{R}_{\mathrm{CM}}\right)^{2}$ is the (squared) hyper radius describing the overall size of the $N$-body system, and $\Omega$ denotes a set of $(3 N-4)$-dimensional hyperangles, describing rotation and deformation degrees of freedom. The hyper-radial equation takes the form

$\left[-\frac{1}{2 m} \frac{d^{2}}{d R^{2}}+\frac{s^{2}-\frac{1}{4}}{2 m R^{2}}+\frac{m \omega^{2} R^{2}}{2}\right] \mathcal{F}_{s}(R)=\left(\mathcal{E}-\mathcal{E}_{\mathrm{CM}}\right) \mathcal{F}_{s}(R)$,

where $\mathcal{E}_{\mathrm{CM}}$ is the energy of the center-of-mass motion, and $s^{2}$ is the hyper-angular eigenvalue obtained by solving the hyper-angular equation. The separability of the hyper-radial and hyper-angular equations originates from the $\mathrm{SO}(2,1)$ dynamical symmetry associated with the scale invariance of the unitary system in an isotropic harmonic trap [12], and it does not generally hold for other confining potentials, e.g., an anisotropic trap or a boxshaped trap [82].

For the equal-mass unitary two-component Fermi system, it is believed that the hyper-angular eigenvalue is positive $s^{2}>0$, and the Efimov effect $[4,83-88]$ does not occur for any number of particles. Though this has not been generally proved, it has been shown numerically for $N_{\uparrow}, N_{\downarrow} \lesssim 5[21,48]$ and mathematically for the $N_{\uparrow}+1$ system with any $N_{\uparrow}$ [89]. Regular solutions of the hyper-radial equation for $s^{2}>0$ are obtained as [49]:

$$
\mathcal{F}_{s}(R)=R^{s+\frac{1}{2}} e^{-\frac{R^{2}}{2 a_{\mathrm{ho}}^{2}}} L_{q}^{(s)}\left(\frac{R^{2}}{a_{\mathrm{ho}}^{2}}\right),
$$

where $L_{q}^{(s)}$ is the generalized Laguerre polynomial, and $q=0,1,2 \ldots$ is a quantum number characterizing the $\mathrm{SO}(2,1)$ ladder of the trapped energy spectrum: $\mathcal{E}-$ $\mathcal{E}_{\mathrm{CM}}=(s+2 q+1) \omega$. We focus on the ground state $q=0$, which simplifies the above wave function since $L_{q=0}^{(s)}=1$.

We note that the wave function and energy of the noninteracting system have the same form as those of the unitary system in Eqs. $(26,28)$. The only difference is the change in the hyper-angular eigenvalue $s \rightarrow s_{0}$, which is related to the non-interacting energy $\mathcal{E}_{\mathrm{NI}}$ via $\mathcal{E}_{\mathrm{NI}}-\mathcal{E}_{\mathrm{CM}}=$ $\left(s_{0}+2 q+1\right) \omega$.

Using separability and the similarity of the wave functions of the unitary and non-interacting systems in the trap, and rewriting $\int \prod_{i=1}^{N} d^{3} \boldsymbol{r}_{i}=$ $\int d^{3} \boldsymbol{R}_{\mathrm{CM}} R^{3 N-4} d R d \Omega$, the squared wave-function overlap between unitary and non-interacting ground states reads

$$
\mathcal{Z}=\frac{\mathcal{I}_{s, s_{0}}^{2}}{\mathcal{I}_{s, s} \mathcal{I}_{s_{0}, s_{0}}} \frac{\left|\left\langle\Phi_{s_{0}} \mid \Phi_{s}\right\rangle\right|^{2}}{\left\langle\Phi_{s_{0}} \mid \Phi_{s_{0}}\right\rangle\left\langle\Phi_{s} \mid \Phi_{s}\right\rangle} .
$$

Here

$$
\mathcal{I}_{s_{1}, s_{2}}=\int d R \mathcal{F}_{s_{1}}(R) \mathcal{F}_{s_{2}}(R)=\frac{a_{\mathrm{ho}}^{s_{1}+s_{2}+2}}{2} \Gamma\left(\frac{s_{1}+s_{2}}{2}+1\right),
$$

$\left\langle\Phi_{s_{1}} \mid \Phi_{s_{2}}\right\rangle=\int d \Omega \Phi_{s_{1}}^{*}(\Omega) \Phi_{s_{2}}(\Omega)$, and we have assumed that the non-interacting and unitary systems are in the same center-of-mass motion state. We note that Eq. (29) holds rigorously for any number of particles, few-body or many-body, and any population imbalance for equalmass two-component Fermi systems. Since the derivation relies only on the separability of the wave function, a similar result can be obtained, with properly defined mass-scaled Jacobi coordinates and hyper-radius [84, 86], for any other scale-invariant few-body or many-body systems [91]

From Eq. (29) we can derive a rigorous upper bound, i.e., $\mathcal{Z}_{>} \geq \mathcal{Z}$, for the squared overlap of the wave functions [92]:

$$
\mathcal{Z}_{>}=\frac{\left[\Gamma\left(\frac{s_{0}+s}{2}+1\right)\right]^{2}}{\Gamma(s+1) \Gamma\left(s_{0}+1\right)} .
$$


Physically, $\mathcal{Z}_{>}$represents the overall spatial overlap of the hyper-radial part of the two wave functions. Indeed, if the energy difference between the non-interacting and unitary systems is large, their sizes, characterized by the $R^{s+\frac{1}{2}}$ term in Eq. (28), become substantially different, rendering $\mathcal{Z}_{>}$small. We can compute the upper bound $\mathcal{Z}_{>}$once we know the energy eigenvalues for the unitary and non-interacting harmonically trapped system. However, note that it totally dismisses few- and many-body correlations appearing in the hyper-angular wave functions.

\section{Uniform systems}

Interestingly, the hyper-angular equation is the same for the harmonically trapped system and the uniform system, so that they possess the same hyper-angular functions and eigenvalues as those of the trapped systems [12]. The hyper-radial and hyper-angular parts are also separable. The only difference between the trapped and uniform systems is that the hyper-radial part $F_{s}(R)$ now satisfies Eq. (27) at $\omega=0$. The hyper-radial wave function which is regular at $R \rightarrow 0$ is then $F_{s}(R) \propto \sqrt{R} J_{s}(k R)$ where $J_{n}$ is the Bessel function, and $k^{2}=2 m\left(E-E_{\mathrm{CM}}\right)$. At zero energy, in particular, it becomes $F_{s}(R)=R^{s+\frac{1}{2}}$.

Similarly to the trapped system, the squared wavefunction overlap for the uniform system at zero energy is

$$
Z=\frac{I_{s, s_{0}}^{2}}{I_{s, s} I_{s_{0}, s_{0}}} \frac{\left|\left\langle\Phi_{s_{0}} \mid \Phi_{s}\right\rangle\right|^{2}}{\left\langle\Phi_{s_{0}} \mid \Phi_{s_{0}}\right\rangle\left\langle\Phi_{s} \mid \Phi_{s}\right\rangle}
$$

where

$$
I_{s_{1}, s_{2}}=\int d R F_{s_{1}}(R) F_{s_{2}}(R)=\int d R R^{s_{1}+s_{2}+1} .
$$

This integral is formally divergent at large $R$ but this is compensated by the same divergence appearing in the denominator in Eq. (32). We thus relate the squared overlap of the unitary and non-interacting ground state wave functions in the uniform $(Z)$ and trapped $(\mathcal{Z})$ systems:

$$
Z=\frac{\Gamma(s+2) \Gamma\left(s_{0}+2\right)}{\left[\Gamma\left(\frac{s_{0}+s}{2}+2\right)\right]^{2}} \mathcal{Z}
$$

Importantly, this exact relation enables us to extract the uniform space result from only the energy (corresponding to $s$ ) and the harmonic trap overlap. We emphasize that the above arguments and results are valid for any number of particles, for both few- and many-body systems, in marked contrast to LDA, which is valid only for systems with large number of particles.

From Eq (34), one also finds the upper limit of $Z$ for the uniform system, $Z_{>} \geq Z$, where

$$
Z_{>}=\frac{4(s+1)\left(s_{0}+1\right)}{\left(s+s_{0}+2\right)^{2}}=\frac{\Gamma(s+2) \Gamma\left(s_{0}+2\right)}{\left[\Gamma\left(\frac{s_{0}+s}{2}+2\right)\right]^{2}} \mathcal{Z}_{>} .
$$

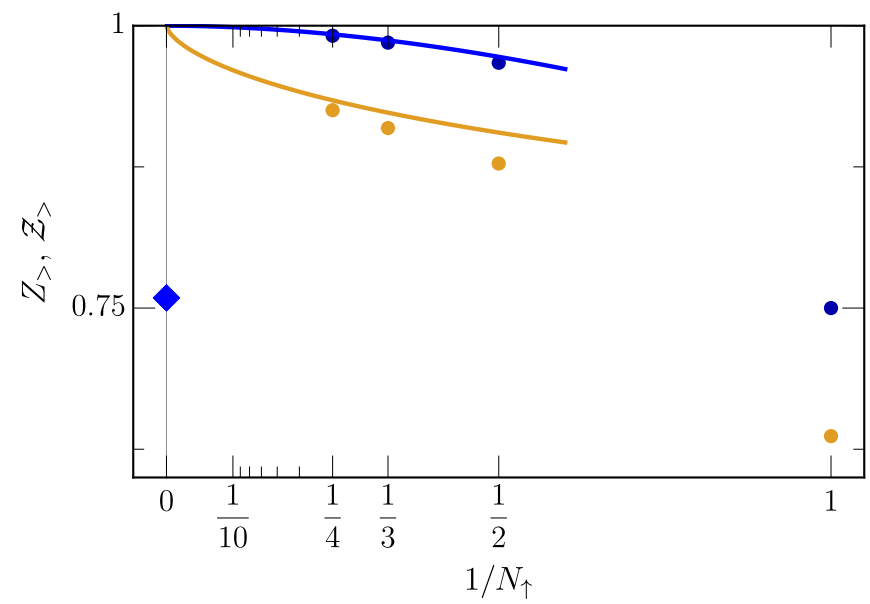

FIG. 5: Upper limit of the polaron residue for the trapped (yellow) and uniform systems (blue), as a function of the number of majority particles $N_{\uparrow}$. The circles represent the few-body results in Eqs. $(31,35)$ evaluated with the energies calculated in Refs. [21, 48, 51]. The error bars are smaller than the symbol sizes. The solid curves are the many-body result in Eqs. (36,37). For $N_{\uparrow} \rightarrow \infty$, the polaron residue in the uniform system $Z_{\text {pol }}=0.759$ [42] is indicated as a blue diamond. For the upper bound curve we have used the constants $m^{*} / m=1.198$ and $A=0.615$ [40-42].

\section{B. Applications to few- and many-body systems}

\section{Polaron residue in the limit of large spin imbalance}

We now apply our hyperspherical approach to evaluate the upper bound for the polaron residue at unitarity. To this end, we use the energy of the trapped $N_{\uparrow}+1$ Fermi system at large $N_{\uparrow}: \mathcal{E}=\mathcal{E}_{0 \uparrow}-A \mu+\frac{3}{2} \omega \sqrt{\frac{m}{m^{*}}(1+A)}$ - see the discussion in Sec. III. Here we take into account the correction to the LDA [29] for both the noninteracting energy, $\mathcal{E}_{0 \uparrow}=\frac{\omega}{8}\left(6 N_{\uparrow}\right)^{4 / 3}\left[1+\frac{1}{2}\left(6 N_{\uparrow}\right)^{-2 / 3}\right]$, and the chemical potential $\mu=\partial \mathcal{E}_{0 \uparrow} / \partial N_{\uparrow}$. From the polaron energy, or rather from $\left(s_{0}+1\right) \omega=\mathcal{E}_{0 \uparrow}-\frac{3}{2} \omega$ and $(s+1) \omega=\mathcal{E}-\frac{3}{2} \omega$, one can evaluate the upper bound of its residue. Using Eq. (31) together with the Stirling formula for the Gamma function, we obtain

$$
\mathcal{Z}_{>}=1-\frac{2^{\frac{1}{3}} A^{2}}{\left(3 N_{\uparrow}\right)^{\frac{2}{3}}}+\frac{A}{N_{\uparrow}}\left[\sqrt{\frac{m}{m^{*}}(1+A)}-1\right]+O\left(N_{\uparrow}^{-\frac{4}{3}}\right)
$$

and

$Z_{>}=1-\frac{4 A^{2}}{9 N_{\uparrow}^{2}}+\frac{2^{\frac{5}{3}} A}{3^{\frac{4}{3}} N_{\uparrow}^{\frac{7}{3}}}\left[\sqrt{\frac{m}{m^{*}}(1+A)}-1\right]+O\left(N_{\uparrow}^{-\frac{8}{3}}\right)$

for the trapped and uniform systems, respectively.

In Fig. 5 we show these $N_{\uparrow} \gg 1$ upper bounds for the polaron (solid lines), together with few-body results (circles) obtained from Eqs. $(31,35)$ and few-body data. We see that these calculations match quite well for intermediate $N_{\uparrow}$. We note that for two-body systems (right-most 
circles), our upper bounds are in fact equal to the exact values in Eq. (25). Quite remarkably, the exact residue of $3 / 4$ from the two-body problem in uniform space is very close to the expected many-body limit of $Z=0.759$ [42]. It will be of interest to determine whether this close agreement between the few- and many-body limits holds for intermediate particle numbers. From Eq. (34), we further conclude that the polaron residues in both the uniform and the trapped systems approach the same value in the many-body limit, which is consistent with the expectation that the impurity is primarily located at the center of the trap.

\section{Unpolarized many-body system}

The energy of the trapped unitary system is characterized by the Bertsch parameter via $\mathcal{E}=2 \sqrt{\xi} \mathcal{E}_{0 \uparrow}$, from which one finds $(s+1) \omega=2 \sqrt{\xi} \mathcal{E}_{0 \uparrow}-\frac{3}{2} \omega$ and $\left(s_{0}+1\right) \omega=2 \mathcal{E}_{0 \uparrow}-\frac{3}{2} \omega$. Substituting these values into Eq. (31), we find the upper bound in the trap

$$
\mathcal{Z}_{>}=\exp \left[-d_{\xi} N^{4 / 3}\left(1+\frac{(3 N)^{-2 / 3}}{2}\right)+O(1)\right]
$$

where

$$
d_{\xi}=\frac{3^{\frac{4}{3}}}{4}\left[(1+\sqrt{\xi}) \log \left(\frac{2}{1+\sqrt{\xi}}\right)+\sqrt{\xi} \log \sqrt{\xi}\right] \simeq 0.0506
$$

and we have used the Bertsch parameter in Eq. (22). Similarly, we find the upper bound for a uniform system

$$
Z_{>}=\frac{4 \sqrt{\xi}}{(1+\sqrt{\xi})^{2}}+O\left(N^{-\frac{4}{3}}\right) \simeq 0.942+O\left(N^{-\frac{4}{3}}\right) .
$$

The upper bounds for the many-body squared overlaps are shown in Fig. 6 as solid lines, together with results from few-body physics (circles). Once again, we see that the few- and many-body physics agree well for intermediate particle numbers. We emphasize that our results are strict upper bounds, and they thus prove that the trapped ground state at unitarity has zero overlap with its non-interacting counterpart in the many-body limit. On the other hand, the upper bound $Z_{>}$remains finite for the uniform system, which implies that the hyperangular component dominates the behaviour of the overlap for large $N$ in this case.

\section{CONCLUSIONS AND OUTLOOK}

In this topical review paper, we have revisited the ground-state properties of the unitary Fermi gas from the perspective of few-body physics. From the energies of unpolarized trapped few-body systems, we found that we could extract values for the Bertsch parameter that all lie within $15 \%$ of that obtained from precision experiments [9]. Indeed, the simplest two-body result only deviated from the established value by $7 \%$, lending weight

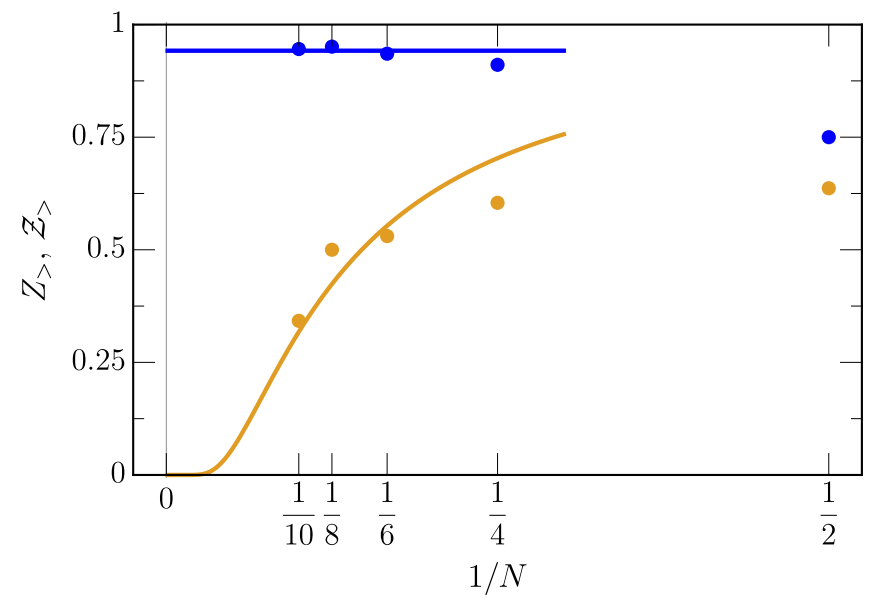

FIG. 6: Upper limit of the squared overlap between the interacting and non-interacting ground states for the spin-balanced system in a trap (yellow) and in uniform space (blue). The circles represent the few-body results in Eqs. (31,35) evaluated with the energies calculated in Refs. [21, 48], and the solid curves are the many-body results in Eqs. $(38,39)$. The error bars resulting from uncertainty in the few-body energies are smaller than the symbol sizes.

to the idea that the Bertsch parameter is primarily determined by two-body correlations. In the limit of large spin imbalance, we observed a similar accuracy for the polaron energy at unitarity, and we determined its dependence on spin- $\uparrow$ particle number for $N_{\uparrow} \gg 1$. Furthermore, we could obtain a reasonable estimate for the polaron's effective mass by fitting the energy as a function of $N_{\uparrow}$.

We also investigated the contact, another key thermodynamic quantity that characterizes the unitary Fermi system. We found that the few-body results for the unpolarized system converged particularly rapidly, with the values for the contact lying well within the range of results determined in the literature for the many-body system. We could also extract a pairing gap from the contact that was consistent with the experimentally determined value [32]. It is thus conceivable that the pairing gap is intimately related to the contact in the unitary ground state.

Finally, we analyzed the squared wave-function overlap between the ground state of the unitary Fermi system and its non-interacting counterpart. Here, we showed that the overlap for the two-body system in uniform space is remarkably close to the predicted polaron residue in the many-body limit. We also derived a rigorous upper bound of the squared overlap for both the trapped and uniform systems. Using this, we proved that the trapped unpolarized ground state at unitarity has zero overlap with the non-interacting state in the many-body limit.

The successful use of few-body systems to infer manybody properties suggests that the unitary equation of state at zero temperature is dominated by few-body correlations. Thus, a natural question is whether this gener- 
ally holds for a range of systems or whether it is specific to the unitary Fermi gas. In particular, is this behavior generic for any scattering length? Are Fermi statistics required and/or is it necessary to have interactions that are sufficiently short ranged? Could the harmonic trapping potential commonly used in few-body calculations be particularly beneficial in minimizing finite-size effects? Finally, it would be interesting to investigate whether there are any other observables at zero temperature (e.g., dynamical quantities) which cannot be captured within the few-body framework.

\section{ACKNOWLEDGEMENTS}

We are grateful to D. Blume for sharing her fewbody data with us. We would also like to thank
D. Blume, C. Bradly, and A. Martin for fruitful discussions. JL, SE, and MMP acknowledge financial support from the Australian Research Council via Discovery Project No. DP160102739. PM acknowledges funding from a "Ramón y Cajal" fellowship, from MINECO (Severo Ochoa SEV-2015-0522, and FOQUS FIS2013-46768), Generalitat de Catalunya (SGR 874 and CERCA), and the Fundació Privada Cellex. This research was supported in part by the National Science Foundation under Grant No. NSF PHY11-25915. All the authors wish to thank the KITP for the generous hospitality during the program "Universality in Few-Body Systems".
[1] S. Giorgini, L. P. Pitaevskii, and S. Stringari, Theory of ultracold atomic Fermi gases, Rev. Mod. Phys. 80, 1215 (2008).

[2] M. M. Parish, The BCS-BEC crossover, in Quantum Gas Experiments - Exploring Many-Body States, edited by P. Törmä and K. Sengstock (Imperial College Press, 2015).

[3] H. Heiselberg, Crossovers in Unitary Fermi Systems, in The BCS-BEC Crossover and the Unitary Fermi Gas, edited by W. Zwerger (Springer, Berlin, Heidelberg, 2012) pp. 49-97.

[4] D. S. Petrov, Three-body problem in Fermi gases with short-range interparticle interaction, Phys. Rev. A 67, 010703 (2003).

[5] D. S. Petrov, C. Salomon, and G. V. Shlyapnikov, Weakly Bound Dimers of Fermionic Atoms, Phys. Rev. Lett. 93, 090404 (2004).

[6] T.-L. Ho, Universal Thermodynamics of Degenerate Quantum Gases in the Unitarity Limit, Phys. Rev. Lett. 92, 090402 (2004).

[7] N. Navon, S. Nascimbène, F. Chevy, and C. Salomon, The equation of state of a low-temperature Fermi gas with tunable interactions, Science 328, 729 (2010).

[8] M. Horikoshi, S. Nakajima, M. Ueda, and T. Mukaiyama, Measurement of Universal Thermodynamic Functions for a Unitary Fermi Gas, Science 327, 442 (2010).

[9] M. J. H. Ku, A. T. Sommer, L. W. Cheuk, and M. W. Zwierlein, Revealing the Superfluid Lambda Transition in the Universal Thermodynamics of a Unitary Fermi Gas, Science 335, 563 (2012).

[10] K. Van Houcke, F. Werner, E. Kozik, N. Prokof'ev, B. Svistunov, M. Ku, A. Sommer, L. Cheuk, A. Schirotzek, and M. Zwierlein, Feynman diagrams versus Fermi-gas Feynman emulator, Nature Physics 8, 366 (2012).

[11] D. T. Son, Vanishing Bulk Viscosities and Conformal Invariance of the Unitary Fermi Gas, Phys. Rev. Lett. 98, 020604 (2007).

[12] Y. Castin and F. Werner, The Unitary Gas and its Symmetry Properties, in The BCS-BEC Crossover and the
Unitary Fermi Gas, edited by W. Zwerger (Springer, Berlin, Heidelberg, 2012) pp. 127-191.

[13] C. Cao, E. Elliott, J. Joseph, H. Wu, J. Petricka, T. Schäfer, and J. E. Thomas, Universal Quantum Viscosity in a Unitary Fermi Gas, Science 331, 58 (2011).

[14] B. Hammond, W. Lester Jr., and P. Reynolds, Monte Carlo Methods in Ab Initio Quantum Chemistry, World Scientific Lecture and Course Notes in Chemistry (World Scientific, 1994).

[15] M. Randeria, W. Zwerger, and M. Zwierlein, The BCS$B E C$ Crossover and the Unitary Fermi Gas, in The BCS$B E C$ Crossover and the Unitary Fermi Gas, edited by W. Zwerger (Springer Berlin Heidelberg, Berlin, Heidelberg, 2012) pp. 1-32.

[16] G. C. Strinati, Pairing Fluctuations Approach to the $B C S-B E C$ Crossover, in The BCS-BEC Crossover and the Unitary Fermi Gas, edited by W. Zwerger (Springer Berlin Heidelberg, Berlin, Heidelberg, 2012) pp. 99-126.

[17] A. Bulgac, M. M. Forbes, and P. Magierski, The Unitary Fermi Gas: From Monte Carlo to Density Functionals, in The BCS-BEC Crossover and the Unitary Fermi Gas, edited by W. Zwerger (Springer Berlin Heidelberg, Berlin, Heidelberg, 2012) pp. 305-373.

[18] F. Chevy and C. Mora, Ultra-cold polarized Fermi gases, Rep. Progr. Phys. 73, 112401 (2010).

[19] P. Massignan, M. Zaccanti, and G. M. Bruun, Polarons, dressed molecules and itinerant ferromagnetism in ultracold Fermi gases, Rep. Progr. Phys. 77, 034401 (2014).

[20] X.-J. Liu, H. Hu, and P. D. Drummond, Virial Expansion for a Strongly Correlated Fermi Gas, Phys. Rev. Lett. 102, 160401 (2009).

[21] D. Rakshit, K. M. Daily, and D. Blume, Natural and unnatural parity states of small trapped equal-mass twocomponent Fermi gases at unitarity and fourth-order virial coefficient, Phys. Rev. A 85, 033634 (2012).

[22] Y. Yan and D. Blume, Path-Integral Monte Carlo Determination of the Fourth-Order Virial Coefficient for a Unitary Two-Component Fermi Gas with Zero-Range Interactions, Phys. Rev. Lett. 116, 230401 (2016).

[23] S. Endo and Y. Castin, The interaction-sensitive states of a trapped two-component ideal Fermi gas and application 
to the virial expansion of the unitary Fermi gas, Journal of Physics A: Mathematical and Theoretical 49, 265301 (2016).

[24] S. Nascimbène, N. Navon, K. J. Jiang, F. Chevy, and C. Salomon, Exploring the thermodynamics of a universal Fermi gas., Nature 463, 1057 (2010).

[25] X.-J. Liu, Virial expansion for a strongly correlated Fermi system and its application to ultracold atomic Fermi gases, Physics Reports 524, 37 (2013).

[26] A. Wenz, G. Zürn, S. Murmann, I. Brouzos, T. Lompe, and S. Jochim, From few to many: observing the formation of a Fermi sea one atom at a time, Science 342, 457 (2013).

[27] G. E. Astrakharchik and I. Brouzos, Trapped onedimensional ideal Fermi gas with a single impurity, Phys. Rev. A 88, 021602 (2013).

[28] T. Grining, M. Tomza, M. Lesiuk, M. Przybytek, M. Musiał, R. Moszynski, M. Lewenstein, and P. Massignan, Crossover between few and many fermions in a harmonic trap, Phys. Rev. A 92, 061601 (2015).

[29] D. Blume, Few-body physics with ultracold atomic and molecular systems in traps, Reports on Progress in Physics 75, 046401 (2012).

[30] G. Valtolina, F. Scazza, A. Amico, A. Burchianti, A. Recati, T. Enss, M. Inguscio, M. Zaccanti, and G. Roati, Evidence for ferromagnetic instability in a repulsive Fermi gas of ultracold atoms, arXiv:1605.07850 (2016).

[31] F. Chevy and C. Salomon, Thermodynamics of Fermi Gases, in The BCS-BEC Crossover and the Unitary Fermi Gas, edited by W. Zwerger (Springer Berlin Heidelberg, Berlin, Heidelberg, 2012) pp. 407-446.

[32] A. Schirotzek, Y.-i. Shin, C. H. Schunck, and W. Ketterle, Determination of the Superfluid Gap in Atomic Fermi Gases by Quasiparticle Spectroscopy, Phys. Rev. Lett. 101, 140403 (2008).

[33] G. M. Bruun and H. Heiselberg, Cooper pairing and single-particle properties of trapped Fermi gases, Phys. Rev. A 65, 053407 (2002).

[34] A. Leggett, Diatomic molecules and cooper pairs, in Modern Trends in the Theory of Condensed Matter, Lecture Notes in Physics, Vol. 115, edited by A. Pȩkalski and J. Przystawa (Springer Berlin Heidelberg, 1980) pp. 1327.

[35] J. Levinsen and M. M. Parish, Strongly interacting twodimensional Fermi gases, in Annual Review of Cold Atoms and Molecules (World Scientific, 2015) pp. 1-75.

[36] C. Lobo, A. Recati, S. Giorgini, and S. Stringari, Normal State of a Polarized Fermi Gas at Unitarity, Phys. Rev. Lett. 97, 200403 (2006).

[37] S. Pilati and S. Giorgini, Phase Separation in a Polarized Fermi Gas at Zero Temperature, Phys. Rev. Lett. 100, 030401 (2008).

[38] F. Chevy, Universal phase diagram of a strongly interacting Fermi gas with unbalanced spin populations, Phys. Rev. A 74, 063628 (2006).

[39] R. Combescot, A. Recati, C. Lobo, and F. Chevy, Normal State of Highly Polarized Fermi Gases: Simple Many-Body Approaches, Phys. Rev. Lett. 98, 180402 (2007).

[40] N. Prokof'ev and B. Svistunov, Fermi-polaron problem: Diagrammatic Monte Carlo method for divergent signalternating series, Phys. Rev. B 77, 020408 (2008).

[41] R. Combescot and S. Giraud, Normal State of Highly Polarized Fermi Gases: Full Many-Body Treatment, Phys.
Rev. Lett. 101, 050404 (2008).

[42] J. Vlietinck, J. Ryckebusch, and K. Van Houcke, Quasiparticle properties of an impurity in a Fermi gas, Phys. Rev. B 87, 115133 (2013).

[43] C. J. Pethick and H. Smith, Bose-Einstein Condensation in Dilute Gases (Cambridge University Press, Cambridge, 2008).

[44] F. Werner and Y. Castin, General relations for quantum gases in two and three dimensions: Two-component fermions, Phys. Rev. A 86, 013626 (2012).

[45] F. Werner, L. Tarruell, and Y. Castin, Number of closedchannel molecules in the BEC-BCS crossover, The European Physical Journal B 68, 401 (2009).

[46] S. Hoinka, M. Lingham, K. Fenech, H. Hu, C. J. Vale, J. E. Drut, and S. Gandolfi, Precise Determination of the Structure Factor and Contact in a Unitary Fermi Gas, Phys. Rev. Lett. 110, 055305 (2013).

[47] T. Busch, B.-G. Englert, K. Rzażewski, and M. Wilkens, Two Cold Atoms in a Harmonic Trap, Foundations of Physics 28, 549 (1998).

[48] X. Y. Yin and D. Blume, Trapped unitary two-component Fermi gases with up to ten particles, Phys. Rev. A 92, 013608 (2015).

[49] F. Werner and Y. Castin, Unitary Quantum Three-Body Problem in a Harmonic Trap, Phys. Rev. Lett. 97, 150401 (2006).

[50] K. M. Daily and D. Blume, Energy spectrum of harmonically trapped two-component Fermi gases: Three- and four-particle problem, Phys. Rev. A 81, 053615 (2010).

[51] D. Blume and K. M. Daily, Few-body resonances of unequal-mass systems with infinite interspecies two-body s-wave scattering length, Phys. Rev. A 82, 063612 (2010).

[52] D. Blume and K. Daily, Trapped two-component Fermi gases with up to six particles: Energetics, structural properties, and molecular condensate fraction, Comptes Rendus Physique 12, 86 (2011).

[53] C. J. Bradly, B. C. Mulkerin, A. M. Martin, and H. M. Quiney, Coupled-pair approach for strongly interacting trapped fermionic atoms, Phys. Rev. A 90, 023626 (2014).

[54] D. Blume, J. von Stecher, and C. H. Greene, Universal Properties of a Trapped Two-Component Fermi Gas at Unitarity, Phys. Rev. Lett. 99, 233201 (2007).

[55] A. Bulgac, Local-density-functional theory for superfluid fermionic systems: The unitary gas, Phys. Rev. A 76, 040502 (2007).

[56] R. Jáuregui, R. Paredes, and G. T. Sánchez, BEC-BCS crossover of a trapped Fermi gas without using the local density approximation, Phys. Rev. A 76, 011604 (2007).

[57] S. Y. Chang and G. F. Bertsch, Unitary Fermi gas in a harmonic trap, Phys. Rev. A 76, 021603 (2007).

[58] J. von Stecher, C. H. Greene, and D. Blume, Energetics and structural properties of trapped two-component Fermi gases, Phys. Rev. A 77, 043619 (2008).

[59] A. L. Zubarev and M. Zoubarev, On the kinetic energy of unitary Fermi gas in a harmonic trap, EPL (Europhysics Letters) 87, 33001 (2009).

[60] A. Nicholson, M. Endres, D. B. Kaplan, and J. W. Lee, Lattice Study of Trapped Fermions at Unitarity, in Proceedings of The XXVIII International Symposium on Lattice Field Theory. June 14-19,2010. Villasimius, Sardinia Italy. (2010) p. 206.

[61] J. Carlson and S. Gandolfi, Predicting energies of small clusters from the inhomogeneous unitary Fermi gas, Phys. Rev. A 90, 011601 (2014). 
[62] D. Blume, Trapped polarized Fermi gas at unitarity, Phys. Rev. A 78, 013635 (2008).

[63] J. Carlson, S. Gandolfi, K. E. Schmidt, and S. Zhang, Auxiliary-field quantum Monte Carlo method for strongly paired fermions, Phys. Rev. A 84, 061602 (2011).

[64] J. Levinsen, P. Massignan, G. M. Bruun, and M. M. Parish, Strong-coupling ansatz for the one-dimensional Fermi gas in a harmonic potential, Science Advances 1, e1500197 (2015).

[65] A. Recati, C. Lobo, and S. Stringari, Role of interactions in spin-polarized atomic Fermi gases at unitarity, Phys. Rev. A 78, 023633 (2008).

[66] S. Tan, Energetics of a strongly correlated Fermi gas, Annals of Physics 323, 2952 (2008).

[67] S. Tan, Large momentum part of a strongly correlated Fermi gas, Annals of Physics 323, 2971 (2008).

[68] E. Braaten, Universal Relations for Fermions with Large Scattering Length, in The BCS-BEC Crossover and the Unitary Fermi Gas, edited by W. Zwerger (Springer Berlin Heidelberg, Berlin, Heidelberg, 2012) pp. 193-231.

[69] Y. Sagi, T. E. Drake, R. Paudel, and D. S. Jin, Measurement of the Homogeneous Contact of a Unitary Fermi Gas, Phys. Rev. Lett. 109, 220402 (2012).

[70] R. Combescot, S. Giorgini, and S. Stringari, Molecular signatures in the structure factor of an interacting Fermi gas, EPL (Europhysics Letters) 75, 695 (2006).

[71] R. Haussmann, M. Punk, and W. Zwerger, Spectral functions and $r f$ response of ultracold fermionic atoms, Phys. Rev. A 80, 063612 (2009).

[72] S. Gandolfi, K. E. Schmidt, and J. Carlson, BEC-BCS crossover and universal relations in unitary Fermi gases, Phys. Rev. A 83, 041601 (2011).

[73] F. Palestini, A. Perali, P. Pieri, and G. C. Strinati, Temperature and coupling dependence of the universal contact intensity for an ultracold Fermi gas, Phys. Rev. A 82, 021605 (2010).

[74] H. Hu, X.-J. Liu, and P. D. Drummond, Universal contact of strongly interacting fermions at finite temperatures, New Journal of Physics 13, 035007 (2011).

[75] Y. Yan and D. Blume, Harmonically trapped Fermi gas: Temperature dependence of the Tan contact, Phys. Rev. A 88, 023616 (2013).

[76] D. Blume, private communication.

[77] M. Punk, P. T. Dumitrescu, and W. Zwerger, Polaronto-molecule transition in a strongly imbalanced Fermi gas, Phys. Rev. A 80, 053605 (2009).

[78] C. Kohstall, M. Zaccanti, M. Jag, A. Trenkwalder, P. Massignan, G. M. Bruun, F. Schreck, and R. Grimm, Metastability and coherence of repulsive polarons in a strongly interacting Fermi mixture, Nature 485, 615 (2012).

[79] M. M. Parish and J. Levinsen, Quantum dynamics of impurities coupled to a Fermi sea, Phys. Rev. B 94, 184303 (2016).

[80] F. Scazza, G. Valtolina, P. Massignan, A. Recati, A. Amico, A. Burchianti, C. Fort, M. Inguscio, M. Zaccanti, and G. Roati, Observation of repulsive Fermi polarons in a resonant mixture of ultracold ${ }^{6} \mathrm{Li}$ atoms,
arXiv:1609.09817 (2016)

[81] A. Schirotzek, C.-H. Wu, A. Sommer, and M. W. Zwierlein, Observation of Fermi Polarons in a Tunable Fermi Liquid of Ultracold Atoms, Phys. Rev. Lett. 102, 230402 (2009).

[82] A. L. Gaunt, T. F. Schmidutz, I. Gotlibovych, R. P. Smith, and Z. Hadzibabic, Bose-Einstein Condensation of Atoms in a Uniform Potential, Phys. Rev. Lett. 110, 200406 (2013).

[83] V. Efimov, Weakly-bound states of three resonantlyinteracting particles, Yad. Fiz. 12, 1080 (1970), [Sov. J. Nucl. Phys. 12, 589-595 (1971)].

[84] E. Braaten and H.-W. Hammer, Universality in fewbody systems with large scattering length, Physics Reports 428, 259 (2006).

[85] F. Ferlaino and R. Grimm, Trend: Forty years of Efimov physics: How a bizarre prediction turned into a hot topic, Physics 3, 9 (2010).

[86] P. Naidon and S. Endo, Efimov Physics: a review, arXiv:1610.09805 (2016).

[87] V. Efimov, Energy levels of three resonantly interacting particles, Nuclear Physics A 210, 157 (1973).

[88] Y. Castin, C. Mora, and L. Pricoupenko, Four-Body Efimov Effect for Three Fermions and a Lighter Particle, Phys. Rev. Lett. 105, 223201 (2010).

[89] T. Moser and R. Seiringer, Stability of a fermionic $N+1$ particle system with point interactions, arXiv:1609.08342 (2016).

[90] In general, we will use calligraphic symbols to denote the trapped thermodynamic observables.

[91] Equation (29) remains valid even when the $N$-body Efimov effect [83-86] occurs, as long as any $n<N$ subsystem does not show the Efimov effect. As examples, it is valid for a system of 3 identical bosons [83], since the 3-body parameter appears only in the hyper-radial wave function. For a mass-imbalanced $2+1$ Fermi system, in which the Efimov effect occurs for $m_{\uparrow} / m_{\downarrow}>$ $13.606 \ldots[4,87]$, it holds for the entire mass ratio. We note however that when a $n<N$ subsystem shows the Efimov effect, the hyper-angular wave function depends on an additional $n$-body parameter, and Eq. (29) breaks down. A prime example would be the mass-imbalanced $3+1$ Fermi system for $m_{\uparrow} / m_{\downarrow}>13.606 \ldots$, where the three-body parameter non-trivially appears in the hyperradial and hyper-angular wave functions, so that the total wave function cannot be written as in the separable form of Eq. (26). For $m_{\uparrow} / m_{\downarrow}<13.606 \ldots$., on the other hand, Eq. (29) is valid, even when the four-body Efimov states appear [88].

[92] An upper bound similar to Eq. (31) can be derived even in the presence of an $N$-body Efimov effect $\left(s^{2}<0\right)$ as long as the Efimov effect does not occur in any subsystem (see the footnote above), by replacing the hyper-radial wave function in Eq. (28) by the corresponding hyperradial solution $\mathcal{F}_{s}(R)=R^{-\frac{1}{2}} W_{\frac{\left(\mathcal{E}-\mathcal{E}_{\mathrm{CM})}\right.}{2 \omega}, \frac{s}{2}}\left(\frac{R^{2}}{a_{\mathrm{ho}}^{2}}\right)$ [49], supplemented with an $N$-body boundary condition at small hyper-radius. Here, $W$ is the Whittaker function. 